\title{
Transnationales linguistisches Kapital der Bürger und der Prozess der europäischen Integration
}

\author{
Jürgen Gerhards
}

Berliner Studien zur Soziologie Europas

Nr. 17

Dezember 2008

Freie Universität Berlin, Institut für Soziologie, Garystraße 55, D-14195 Berlin 
Die „Berliner Studien zur Soziologie Europas“ des Lehrstuhls für Makrosoziologie der Freien Universität Berlin verstehen sich als ein Ort zur Vorpublikation von Beiträgen, die später in Fachzeitschriften und Sammelbänden veröffentlicht werden sollen. Die Beiträge sollen helfen, eine Soziologie Europas zu profilieren; sie stehen auch im Kontext des Master-Studiengangs „Soziologie - Europäische Gesellschaften“.

Gegenstand der Reihe sind Beiträge zur Analyse der Herausbildung einer europäischen Gesellschaftsstruktur und -kultur, vergleichende Analysen, die die Unterschiede und Gemeinsamkeiten zwischen verschiedenen europäischen Gesellschaften thematisieren, sowie theoretische Versuche einer Soziologie Europas.

Ziel der Reihe ist es, durch die frühe Verbreitung dieser Arbeiten den wissenschaftlichen Gedankenaustausch zu fördern. Die Beiträge sind nur über das Internet als pdf-Datei zu beziehen.

Zitationsweise: BSSE-Arbeitspapier Nr.17. Berlin: Freie Universität Berlin.

Dieser Artikel erscheint in: Monika Eigmüller und Steffen Mau (Hg.) (2009): Gesellschaftstheorie und Europapolitik. Sozialwissenschaftliche Ansätze zur Europaforschung. Wiesbaden: VS Verlag. 


\begin{abstract}
Der politische Prozess der europäischen Integration hat entscheidend zu einer Europäisierung der Gesellschaften Europas beigetragen. Ob dieser systemische Prozess der Europäisierung von einer lebensweltlichen Europäisierung im Sinne einer Zunahme der Kommunikation zwischen den Bürgern verschiedener Mitgliedsländer begleitet wird, hängt entscheidend von der Mehrsprachigkeit der Bürger ab. In welchem Maße die Bürger der 27 Länder mehrsprachig sind und wie man die Unterschiede in der Ausstattung mit transnationalem, linguistischem Kapital erklären kann, ist das Thema des Artikels. In einem ersten Schritt werde ich die Sprachpolitik der Europäischen Union beschreiben. Diese ist durch zwei Merkmale gekennzeichnet: Die Akzeptanz der sprachlichen Heterogenität der Europäischen Union und der Verzicht auf eine Politik der sprachlichen Homogenisierung einerseits und die Förderung der Mehrsprachigkeit der Bürger Europas zur Verbesserung der Integration Europas andererseits. Im zweiten Schritt analysiere ich auf der Basis einer Auswertung einer Eurobarometerbefragung, über welches transnationale linguistische Kapital die Bürger Europas verfügen, das sie in die Lage versetzt, am Europäisierungsprozess zu partizipieren. Die Ergebnisse werden zeigen, dass die Kompetenzen der Mehrsprachigkeit zwischen den Ländern und innerhalb der Länder sehr unterschiedlich verteilt sind, es ähnlich wie bei anderen Kapitalien eine Ungleichheit in der Kapitalausstattung der Bürger gibt. Im dritten Schritt gehe ich der Frage nach, wie man diese Ungleichheit erklären kann. Dazu werden zuerst einige Hypothesen formuliert, die dann durch eine multivariate Analyse überprüft werden. Die Mehrsprachigkeit der Bürger kann durch folgende Faktoren relativ gut erklärt werden: den Grad der Modernität und die Größe eines Landes, in dem jemand lebt, die Verfügung über institutionalisiertes kulturelles Kapital und durch die Klassenlage des Befragten. Dieser Befund bestätigt andere Ergebnisse, die gezeigt haben, dass es vor allem die oberen Schichten sind, die vom Prozess der systemischen europäischen Integration profitieren, weil sie in der Lage sind, daran zu partizipieren.
\end{abstract}




\section{Transnationales linguistisches Kapital der Bürger und der Prozess der europäi- schen Integration ${ }^{1}$}

Die Entwicklung der Europäischen Union ist seit ihrer Gründung neben einer Ausdehnung des territorialen Herrschaftsbereichs durch die Aufnahme neuer Mitglieder durch eine zunehmende Vertiefung gekennzeichnet. Die damalige Europäische Gemeinschaft startete bekanntlich mit der Festlegung einer gemeinsamen Verwaltung für die Kohle- und Stahlindustrie, institutionalisiert durch die Montanunion. Schritt für Schritt wurden andere, zunächst nur wirtschaftliche Bereiche in den Prozess der Vertiefung einbezogen: Eine Zollunion wurde gegründet, ein gemeinsamer Binnenmarkt und eine Wirtschafts- und Währungsunion wurden gebildet und für eine Teilgruppe der EU-Länder wurde eine gemeinsame Währung eingeführt. Mit dem Vertrag von Maastricht wurden die Handlungsfelder europäischer Politik über die engeren wirtschaftlichen Bereiche hinaus auf die Bereiche Außen-, Innen- und Sicherheitspolitik ausgedehnt. Die Ausdehnung der Handlungsfelder europäischer Politik findet ihr institutionelles Pendant in der Entwicklung und Ausdehnung einer europäischen Institutionenordnung, eines eigenständigen, zunehmend mehr Aufgaben übernehmenden Herrschaftsverbandes. Dazu haben die Staaten der EU einen Teil der nationalen Souveränitätskompetenzen auf die EU übertragen; die Nationalstaaten und ihre Bürger sind den Beschlüssen der EU unmittelbar unterworfen, Europarecht bricht nationales Recht; die Kommission überwacht die Implementierung der Beschlüsse und der Europäische Gerichtshof kann die Mitgliedsstaaten bei Nichtbefolgung sanktionieren (Lepsius 1990).

Der politische Integrationsprozess hat eine Europäisierung der Mitgliedsländer der EU bewirkt. Unter Europäisierung verstehe ich einen Spezialfall von Transnationalisierungsprozessen. Transnationalisierung wiederum haben wir an früherer Stelle in Aufnahme von Überlegungen von Karl W. Deutsch (1953) als die Zunahme von Austauschprozessen, die über die nationalstaatlichen Grenzen hinausreichen, definiert (vgl. Gerhards und Rössel 1999; vgl. auch de Swaan 1995; Pries 2008); andere Autoren sprechen von Denationalisierung und meinen Ähnliches (vgl. Beisheim, Dreher, Walter und Zürn 1999; Zürn 1998). Dehnen sich Austauschprozesse unterschiedlicher Art über die nationalstaatlichen Grenzen aus und finden sie in erster Linie innerhalb Europas statt, kann man von Europäisierungsprozessen sprechen (Gerhards und Rössel 1999; ähnlich Delhey 2005). Empirisch gibt es nun eine Vielzahl von Hinweisen, dass der politische Integrationsprozess zu einer Europäisierung der Gesellschaften Europas beigetragen hat (aus historischer Perspektive vgl. Kaelble 2005, 2007). Vor allem die Herstellung des europäischen Binnenmarkts sowie die damit verbundene Freizügigkeit für Waren, Kapital, Dienstleistungen und Arbeits-

\footnotetext{
${ }^{1}$ Ich bedanke mich bei Inga Ganzer, David Glowsky und Mike S. Schäfer für eine Kommentierung des Artikels, bei Monika Hufnagel für die Unterstützung bei der Datenanalyse.
} 
kräfte hat grenzüberschreitende Wirtschaftsprozesse befördert und entscheidend das Anwachsen des binneneuropäischen Handels, die Zunahme grenzüberschreitender und vor allem europäischer Wertschöpfungsketten, die Transnationalisierung des Kapitals und eine innereuropäische Migration begünstigt (vgl. z. B. Ambrosius 1996; Hirst und Thompson 1998; Fligstein und Stone Sweet 2002; Fligstein und Merand 2002; Verwiebe 2004). Ob dieser systemische Prozess der Europäisierung aber von einer lebensweltlichen Europäisierung begleitet und somit die Europäisierung von oben durch eine Europäisierung von unten (Mau 2007: 53) ergänzt wird, ist erst jüngst in den Aufmerksamkeitsfokus der Forschung geraten (vgl. Mau und Mewes 2007; Mau 2007; Delhey 2004, 2005).

Georg Vobruba, der zu einer Profilierung einer genuin soziologischen Europaforschung beigetragen hat (Vobruba 2001, 2005) geht in einem programmatischen Aufsatz zur Bestimmung der Aufgaben einer Europasoziologie davon aus, dass die Analyse des Spannungsverhältnisses zwischen der institutionellen europäischen Ordnung auf der einen Seite und der sozialen Integration Europas auf der anderen Seite zu den zentralen Aufgaben einer sich entwickelten Europasoziologie gehört (Vobruba 2008). Genau an diese Problemstellung knüpfen die folgenden Überlegungen an. Der Prozess der lebensweltlichen Integration im Sinne einer Zunahme der Kommunikation zwischen den Bürgern verschiedener Mitgliedsländer, in denen bekanntlich unterschiedliche Sprachen gesprochen werden, wird entscheidend begünstigt, wenn die Bürger mehrere Sprachen beherrschen. In welchem Maße dies der Fall ist und wie man die Unterschiede in der Ausstattung mit transnationalem, linguistischem Kapital - der Begriff wird an späterer Stelle mit Rückgriff auf die Theorie Bourdieus erläutert werden - erklären kann, ist das Thema der folgenden Ausführungen. In einem ersten Schritt werde ich die Sprachpolitik der Europäischen Union beschreiben. Diese ist durch zwei Merkmale gekennzeichnet: Die Akzeptanz der sprachlichen Heterogenität der Europäischen Union und der Verzicht auf eine Politik der sprachlichen Homogenisierung einerseits und die Förderung der Mehrsprachigkeit der Bürger Europas zur Verbesserung der Integration Europas andererseits. Im zweiten Schritt analysiere ich auf der Basis einer Auswertung einer Eurobarometerbefragung, über welches linguistische Kapital die Bürger Europas verfügen, das sie in die Lage versetzt, am Europäisierungsprozess zu partizipieren. Die Ergebnisse werden zeigen, dass die Kompetenzen der Mehrsprachigkeit zwischen den Ländern und innerhalb der Länder sehr unterschiedlich verteilt sind, es ähnlich wie bei anderen Kapitalien eine Ungleichheit in der Kapitalausstattung der Bürger gibt. Im dritten Schritt gehe ich der Frage nach, wie man diese Ungleichheit erklären kann. Dazu werden zuerst einige Hypothesen formuliert, die dann durch eine multivariate Analyse überprüft werden. Die Mehrsprachigkeit der Bürger kann durch folgende Faktoren relativ gut erklärt werden: den Grad der Modernität und die Größe eines Landes, in dem jemand lebt, die Verfügung über institutionalisiertes kulturelles Kapital und durch die Klassenlage des Befragten. Dieser Befund bestätigt andere Ergebnisse, die gezeigt haben, dass es vor allem die oberen Schichten sind, die vom Prozess der systemi- 
schen europäischen Integration profitieren, weil sie in der Lage sind, daran zu partizipieren.

\section{Die Sprachkonstellation Europas und die Sprachpolitik der Europäischen Union}

\subsection{Die Sprachkonstellation Europas}

Eine Sprache besteht im Wesentlichen aus einer Grammatik und aus einem Wortschatz. Personen bezeichnen mit Sätzen, die sie aus dem Wortschatz und mit Hilfe der Grammatik bilden, Dinge in der Welt, bringen Handlungsabsichten zum Ausdruck und äußern über Sprache ihre Gefühle. Andere Personen, die die gleiche Sprache sprechen, verstehen die Bedeutungen der Aussagen und können auf dieser Basis wiederum ihre Äußerungen tätigen, die dann wiederum von dem anderen Interaktionspartner verstanden werden. Über diesen wechselseitigen Austausch von Symbolen können Menschen ihre Handlungsabsichten koordinieren und miteinander kooperieren. Voraussetzung für eine sprachliche Koordination von Interaktionen ist, dass die Sprecher die gleiche Sprache sprechen, also über das gleiche Lexikon und die gleiche Grammatik verfügen. Die einzelnen insgesamt auf der Erde existierenden Sprachen unterscheiden sich von einander durch verschiedene Grammatiken und unterschiedliche Lexika. Einzelsprachen sind entsprechend dadurch gekennzeichnet, dass Sprecher von zwei verschiedenen Einzelsprachen sich nicht bzw. nur mit sehr großem Aufwand verständigen können. Die Tatsache, dass Akteure nicht über eine gemeinsame Sprache verfügen, bedeutet nicht, dass sie nicht miteinander interagieren können. Die Transaktionskosten der Interaktion sind aber im Vergleich zu einer Verfügung über eine gemeinsame Sprache enorm hoch. Jeder, der in einem Land war, dessen Sprache er nicht spricht und versucht hat, den Weg zum Bahnhof oder zum Flughafen herauszufinden, weiß dies. ${ }^{2}$

Die Menge der Einzelsprachen, die auf der Erde gesprochen werden, die Dominanz mancher Sprachen und die Bedeutungslosigkeit anderer Sprachen wird entscheidend durch die Machtstrukturen der gesellschaftlichen Institutionenordnung geprägt (de Swaan 1993, 2001b). So ist zum Beispiel die Dominanz des Englischen in der Welt u.a. das Resultat der Dominanz englischsprachiger Kolonialreiche und der Dominanz der USA in der internationalen Ordnung nach dem 2. Weltkrieg (Crystal 1998; Trabant 2008). Insofern ist die Sprachkonstellation immer auch ein Spiegelbild der hierarchisch strukturierten Weltordnung. Dies gilt auch für die Sprachkonstellation in Europa.

\footnotetext{
${ }^{2}$ Die These, dass eine Verständigung über die Grenzen von Sprachen und Kulturen hinweg und insofern auch die Entstehung einer europäischen Öffentlichkeit möglich ist, wird von Cathleen Kantner (2004) mit grundlagentheoretischen hermeneutischen Argumenten gut begründet. Die grundsätzliche Verständigungsmöglichkeit sagt aber noch nichts über die empirische Verständigungswahrscheinlichkeit aus. Diese wird entscheidend von der Tatsache beeinflusst, ob zwei Sprecher ein und dieselbe Sprache sprechen.
} 
Die Menge der Einzelsprachen, die in Europa der 27 Mitgliedsländer gesprochen werden, ist das Resultat eines langen historischen Prozesses, der eng verknüpft ist mit der Entstehung der Nationalstaaten in Europa (zum Folgenden vgl. Haarmann 1993). Die Entstehung der europäischen Nationalstaaten geht in fast allen Fällen einher mit einer Politik der sprachlichen Homogenisierung, der Ausbildung einer Einheitssprache also, die dann auch zur Amtsprache wird, bei gleichzeitiger Verdrängung und Unterdrückung der Minderheitensprache (vgl. Mann 2001). Einige der kleineren Staaten Europas - z.B. Belgien und die Schweiz - bilden dabei eine Ausnahme von diesem allgemeinen Muster; hier wurde die interne sprachliche Spaltung gesellschaftlich institutionalisiert und hat zu einem versäulten und föderalen Institutionensystem insgesamt geführt, wie Stein Rokkan herausgearbeitet hat (Rokkan 1999). Der zentrale Transmissionsriemen für die Politik der sprachlichen Homogenisierung der Nationalstaaten bildet die Etablierung eines nationalen Schulwesens und die Inklusion aller Bürger in die Schule durch die allgemeine Schulpflicht.

Nationalstaatliche Sprachenpolitik ist aber nicht nur ein Instrument einer nationalstaatlichen Vergesellschaftung gewesen, sondern meist auch ein Medium der Vergemeinschaftung. Die Einheitssprache wird nicht nur zur Amtssprache, sondern auch zur Nationalsprache, zum zentralen Merkmal der Identitätskonstruktion. Fast alle Nationalstaaten haben Institutionen der Sprachpflege entwickelt, regeln den verbindlichen Sprachgebrauch durch den Aufbau eines Wörterbuchs und von Überwachungseinrichtungen der Sprachkontrolle. Sie beginnen, die in ihrer Sprache gesprochene Nationalliteratur zu kanonisieren, pflegen Volkslied und Volkskultur und beschreiben ihre Identität u.a. durch die jeweilige Nationalsprache. All dies ist hinreichend gut von Historikern beschrieben worden, ist damit aber kein historisches Relikt, sondern bis heute wirkungsmächtig, wenn man sich die Sprachkonstellation in Europa anschaut.

Die Europäische Union ist ein Zusammenschluss von 27 Nationalstaaten. Nirgendwo sonst zeigt sich der Staatenbundcharakter so deutlich wie in der Sprachkonstellation. In 18 von 27 Ländern der EU gibt es nur eine Amtssprache; daneben gibt es 6 Länder, die eine Amtssprache haben, aber für bestimmte, kleine Regionen eine zweite Amtssprache zulassen; dazu gehören Österreich, Spanien, Italien, Finnland, die Slowakei und die Niederlande. Die dritte Gruppe bilden die wenigen Länder, die flächendeckend zwei oder mehrere Amtssprachen zulassen, dazu gehören Luxemburg, Belgien und Malta. Die 27 nationalstaatlichen Container der EU, die durch eine je spezifische Institutionenordnung gekennzeichnet sind, verfügen in den meisten Fällen auch über eine einheitliche Sprache. Die Grenze der nationalstaatlichen Institutionenordnung ist damit sehr häufig identisch mit einer eigenständigen Sprache, was der Sprachenordnung insgesamt eine ganz besondere Festigkeit verleiht. 


\subsection{Die Sprachpolitik der Europäischen Union}

Diese segmentär, nach Nationalstaaten differenzierte Sprachenkonfiguration bildet auch die Ausgangskonstellation für die Sprachenpolitik der EU, die durch zwei Merkmale gekennzeichnet ist: Im Unterschied zu den Nationalstaaten betreibt die Europäische Union keine Politik der sprachlichen Homogenisierung der Mitgliedsländer durch Förderung einer einheitlichen „lingua franca“, die dann für die Europäische Union verbindlich wäre. Die EU akzeptiert und fördert sogar die sprachliche Heterogenität Europas (1). Zugleich fördert die Europäische Union durch eine Vielzahl von Programmen die Mehrsprachigkeit der Bürger Europas zur Verbesserung der Integration Europas (2).

(1) Während die EU in vielen Politikbereichen auf eine Homogenisierung und Konvergenz der Mitgliedsländer drängt - ein einheitlicher Markt, eine einheitliche Währung, eine Vereinheitlichung des Rechts etc. - gilt dies für die Sprachenpolitik gerade nicht. Die Akzeptanz der Vielsprachigkeit der EU ist vertragsrechtlich eindeutig verbrieft. Grundlage ist die Verordnung Nr. 1 zur Regelung der Sprachenfrage für die Europäische Wirtschaftsgemeinschaft (EWG 1958), die 1958 vom Rat festgelegt und später um die Sprachen der neuen Mitgliedsländer erweitert wurde. Aufgrund von Artikel 22 der im Jahr 2000 verabschiedeten Charta der Grundrechte der EU muss die Union die sprachliche Vielfalt respektieren; laut Artikel 21 ist jegliche Diskriminierung aus sprachlichen Gründen verboten. Die Europäische Union stützt damit die Gleichberechtigung der verschiedenen Nationalsprachen und hat bis dato keine Anstalten unternommen, auf eine europäische Einheitssprache hinzuwirken. Die Mitgliedsländer würden dies auch nicht akzeptieren. Der Bestand der Einzelsprachen ist nicht nur rechtlich verbrieft, die jeweilige Sprache ist Teil der nationalen Identität des jeweiligen Mitgliedslandes und damit ein Kernbestand seiner Souveränität, die sie nicht aufgeben.

Die Gleichbehandlung aller europäischen (Amts-)Sprachen ist damit das erste und zentrale Ziel der EU in der Sprachpolitik. Sämtliche nationale Amtsprachen der Mitgliedsländer sind zugleich auch die Amtssprachen der EU. Eine Ausnahme bildet hierbei das Luxemburgische, da die Regierung des Landes freiwillig darauf verzichtet hat, Luxemburgisch zur Amtssprache der EU zu machen. Insgesamt gibt es damit 23 Amtssprachen bei 27 Mitgliedsländern: Bulgarisch, Dänisch, Deutsch, Englisch, Estnisch, Finnisch, Französisch, Griechisch, Irisch, Italienisch, Lettisch, Litauisch, Maltesisch, Niederländisch, Polnisch, Portugiesisch, Rumänisch, Schwedisch, Slowakisch, Slowenisch, Spanisch, Tschechisch und Ungarisch. Da manche Sprachen Amtssprachen in zwei Ländern zugleich sind (Flämisch in den Niederlanden und in Belgien, Französisch in Frankreich und in Belgien, Deutsch in Österreich und in Deutschland, Griechisch auf Zypern und in Griechenland), ist die Anzahl der Amtssprachen nicht identisch mit der der Mitgliedsländer. Die aus Nationalstaaten gebildete EU bleibt damit bezüglich ihrer sprachlichen Konfiguration im hohen Maße segmentär differenziert. 
Die Akzeptanz der Amtssprachen der Mitgliedsländer als Amtssprachen der EU und der Verzicht auf eine Politik zur Förderung einer "lingua franca“ ist mit entsprechenden Folgen für die Kommunikation innerhalb der Institutionen der EU verbunden: Alle Gesetze, Dokumente und Verordnungen müssen zugleich in allen 23 Amtssprachen abgefasst werden; und auch die Bürger und die Nationalstaaten können sich in ihrer Sprache an die EU wenden und haben das Recht, in ihrer Sprache eine Antwort zu erhalten; auch die Informationsseiten der EU sind in allen Amtssprachen verfasst.

Über die Arbeitssprachen, die die Institutionen intern verwenden, entscheiden die jeweiligen Geschäftsordnungen (Schumann-Hitlzer und Ostarek 2005: 21). Im Folgenden beschreibe ich kurz, wie die einzelnen Institutionen die Sprachvielfalt handhaben. Man bekommt darüber einen Eindruck, mit welchen gewaltigen Transaktionskosten das Prinzip der Vielsprachigkeit verbunden ist. Die Sprachdienstleistungen der EU-Institutionen kosten die EU ca. 1,1 Mrd. Euro pro Jahr (Generaldirektion Übersetzung 2008).

EU-Kommission: Seit 2001 gebraucht die Kommission Deutsch, Englisch und Französisch als interne Arbeitssprachen (Peterson und Shackleton 2006: 61), wobei Englisch und Französisch bevorzugt werden (Ozvalda 2005: 66f., Schumann-Hitzler und Ostarek 2005: 21). Erst wenn Entscheidungsprozesse abgeschlossen sind, erfolgt die Übersetzung in die anderen Amtssprachen (ebd.). Die Generaldirektionen Übersetzung und Dolmetschen der Kommission sind für die Kommission und die anderen Organe der EU, außer dem Parlament und dem Gerichtshof, zuständig (Mickel und Bergmann 2005: 33). Allein die Generaldirektion Übersetzung beschäftigt 2.350 Mitarbeiter und hat ein jährliches Budget von etwa 280 Mio. Euro (Generaldirektion Übersetzung 2008). Sie ist damit der größte Übersetzungsdienst der Welt. Rund $15 \%$ aller Bediensteten der Kommission gehören zum Sprachendienst (Mickel und Bergmann 2005: 33). Um die Übersetzungsdienste der Institutionen zu entlasten, wurde 1994 das Übersetzungszentrum für die Einrichtungen der Europäischen Union (CdT) eingerichtet.

Der Europäische Rat: In allen Sitzungen des Europäischen Rates wird aus und in alle Amtssprachen übersetzt. Alle Dokumente werden dem Rat ebenfalls in allen Amtssprachen vorgelegt (Mickel und Bergmann 2005: 32).

Der Rat der Europäischen Union: Alle Dokumente, über die der Europäische Rat berät, werden in alle Amtssprachen der EU übersetzt. Bei den Sitzungen des Rats wird aus und in alle Sprachen gedolmetscht (vgl. Homepage des Rats der Europäischen Union). Bei informellen Treffen scheint sich aber die „3+1-Lösung“ (Englisch, Französisch, Deutsch + Sprache der Ratspräsidentschaft) durchzusetzen (Ozvalda 2005). Im Ausschuss der Ständigen Vertreter (ASTV) gilt das Drei-Sprachen-Regime (Englisch, Französisch, Deutsch). In den Ratsarbeitsgruppen der Fachbeamten wird in alle 
Amtssprachen übersetzt, wenn ein Gesetz vorbereitet wird. In der überwiegenden Zahl der Gruppen werden nur fünf EU-Sprachen gedolmetscht (Englisch, Französisch, Deutsch, Spanisch und Italienisch). In 45 Gruppen wird auf Dolmetschung ganz verzichtet und nur Englisch und/oder Französisch gesprochen. Für die Ratsarbeitsgruppen wird das sog. „Marktmodell“ angewandt, d.h. dass sich die Mitgliedsstaaten im Einzelfall für oder gegen eine eigene Übersetzung aussprechen, sich aber an den Kosten mitbeteiligen (Mickel und Bergmann 2005: 32). Während der Rat der Europäischen Union für das Dolmetschen auf die Generaldirektion Dolmetschen zurückgreift, verfügt er auch über einen eigenen Sprachendienst des Generalsekretariats des Rates mit mehr als 700 Übersetzern.

Der Europäische Gerichtshof: Bei Klagen vor dem EuGH ist die Sprache der Klageschrift Verfahrenssprache, soweit es sich um eine der EU-Amtssprachen handelt. In Vorabentscheidungssachen wird die Sprache des nationalen Gerichts, das den Gerichtshof anruft, zur Verfahrenssprache. In den Sitzungen werden die Verhandlungen je nach Bedarf in verschiedene Amtssprachen der Europäischen Union simultan übersetzt (Website des EuGH). Als Arbeitssprache wird allein Französisch verwendet (Weidenfeld 2006: 408). Der EuGH verfügt über eine Direktion Dolmetschen und eine Direktion Übersetzung. Anfang 2006 gehörten der Direktion Übersetzung des Gerichtshofes 796 Mitarbeiter an, die alle mindestens drei Sprachen sprechen und voll ausgebildete Juristen sein müssen. Dies entspricht ca. $45 \%$ des Personals des Gerichtshofs.

Das Europäische Parlament: Die Geschäftsordnung des Europäischen Parlaments legt fest, dass alle Schriftstücke des Parlaments in allen Amtssprachen verfasst werden. Weiterhin haben alle Parlamentsmitglieder das Recht, in ihrer (Amts-)Sprache zu sprechen und alle anderen Amtssprachen in diese Sprache simultan gedolmetscht zu bekommen. Dies gilt ebenso für die Amtssprachen der Anwesenden in Ausschuss- und Delegationssitzungen, dies kann jedoch unter Einverständnis der Mitglieder in Ausnahmefällen umgangen werden (Art. 138 Geschäftsordnung des Europäischen Parlaments). Das Europäische Parlament hat sich in besonderer Weise der Vielsprachigkeit verschrieben. Auf der Homepage wird dazu erklärt: „In Bezug auf das Dolmetschen unterscheidet sich das Europäische Parlament von den anderen Organen der EU dadurch, dass in der täglichen Arbeit das Prinzip der umfassenden kontrollierten Mehrsprachigkeit eingehalten wird“. Um Engpässe in der Übersetzung zu umgehen, hat das Europäische Parlament ein System von „Relais-Sprachen“ eingeführt, d.h. dass „Texte zunächst in die gebräuchlichsten Sprachen übersetzt werden (Englisch, Französisch oder Deutsch). Längerfristig könnten noch weitere Sprachen der Gemeinschaft (Spanisch, Italienisch und Polnisch) ebenfalls zu RelaisSprachen werden." Das Parlament beschäftigt einen eigenen Übersetzungsdienst mit ca. 700 Übersetzern. 
(2) Bildet die Akzeptanz und die Unterstützung der Vielsprachigkeit das erste Ziel der EU-Sprachpolitik, dann ist das Prinzip der Förderung der Mehrsprachigkeit der Bürger Europas das zweite zentrale Ziel. Jeder Bürger, so die Vorstellung, soll neben seiner Muttersprache zwei in der EU gesprochene Sprachen sprechen. Seit 2007 gibt es in der Kommission einen eigenen Kommissar für Mehrsprachigkeit. Welche weitergehenden Ziele verfolgt die EU mit ihrer Politik der Förderung der Mehrsprachigkeit (a) und mit welchen Maßnahmen versucht sie diese zu erreichen (b)?

(a) Das folgende Zitat, das der Website des Kommissars für Mehrsprachigkeit entnommen ist, bringt die Ziele, die sich in vielen rechtlichen Dokumenten der EU ähnlich formuliert finden, recht gut zum Ausdruck: „Die Fähigkeit, in mehreren Sprachen zu kommunizieren, ist ein großer Vorteil für Menschen, doch auch für Organisationen und Unternehmen. Sie verstärkt die Kreativität, überwindet kulturelle Vorurteile, fördert das Denken abseits der ausgetretenen Pfade und kann bei der Entwicklung innovativer Produkte und Dienstleistungen helfen. All diese Fähigkeiten und Tätigkeiten sind volkswirtschaftlich wertvoll. Mehrsprachigkeit macht Menschen auch mobiler, so dass sie leichter in anderen Ländern Ausbildungsmöglichkeiten oder eine Arbeitsstelle suchen können. Sie ist gut für den Einzelnen, die Unternehmen und die Wettbewerbsfähigkeit, und damit auch entscheidend für die Verwirklichung des übergeordneten Ziels der Europäischen Union - die LissabonStrategie zur Schaffung von Arbeitsplätzen und zur Förderung des Wirtschaftswachstums. [...] Ziel der Kommissionspolitik zur Mehrsprachigkeit ist es, diese Stärken miteinander zu verknüpfen. Insbesondere hat sie zum Ziel, das Sprachenlernen und die Sprachenvielfalt in der Gesellschaft zu fördern, eine gesunde, mehrsprachige Wirtschaft zu fördern und den Bürgerinnen und Bürgern in ihrer eigenen Sprache Zugang zu den Rechtsvorschriften der Europäischen Union zu geben. ${ }^{3}$

Die Legitimation einer Politik der Förderung der Mehrsprachigkeit speist sich, wie das Zitat zeigt, aus zwei Motiven, einem kulturellen und einem wirtschaftspolitischen. Die Union möchte zum einen durch die Mehrsprachigkeit ihrer Bürger zur Verbesserung der Verständigung zwischen den Menschen und zum Abbau von Vorurteilen beitragen; sie begreift die Mehrsprachigkeit zum zweiten als eine Investition in die Verbesserung des Humankapitals der Bürger, das diese in die Lage versetzt, auf dem europäischen Markt mobil zu sein und ihre Arbeitskraft auch außerhalb des Nationalstaates einsetzen zu können und damit einen Beitrag für das wirtschaftliche Wachstum Europas insgesamt zu leisten. Mit der Sprachpolitik verhält es sich wie mit vielen anderen Politikbereichen der Europäischen Union: Die EU ist in erster Linie eine Wirtschaftsunion; die Legitimationsbeschaffung für nicht-ökonomische Politikfelder erfolgt in der Regel mit ökonomischen Argumenten (vgl. für die Geschlechter- und Familienpolitik Gerhards, Schäfer und Kämpfer 2009; für die Umweltpolitik Gerhards und Lengfeld 2008).

\footnotetext{
${ }^{3}$ http://ec.europa.eu/commission_barroso/orban/policies/policies_de.htm; Zugriff am 20.11.2008.
} 
(b) Die Maßnahmen, die die EU zur Förderung der Mehrsprachigkeit ihrer Bürger ergriffen hat, sind vielfältig und in aller Regel eingebunden in die Maßnahmen zur Bildungspolitik der EU im Allgemeinen. Dabei waren "Sokrates“ und „Leonardo da Vinci“ die wichtigsten Programme, die 2007 in das „Programm für Lebenslanges Lernen" integriert wurden. Hierbei handelt es sich um ein Aktionsprogramm zur Förderung der transnationalen Zusammenarbeit im Bildungsbereich. Die EU hat für das Programm von 2007 bis 2013 ca. 7 Mrd. Euro bewilligt; es integriert folgende Einzelprogramme (vgl. zum folgenden Generaldirektion Bildung und Kultur 2008): COMENIUS richtet sich an vorschulische Einrichtungen und Schulen bis zum Ende des Sekundarbereichs II sowie an Einrichtungen der Schulverwaltung und der Lehreraus- und -fortbildung. Comenius unterstützt die Mobilität von Schülern, Lehramtsstudierenden und Lehrkräften und fördert damit das Erlernen moderner Fremdsprachen. Gefördert werden Schulpartnerschaften und die Mobilität von Schülern, Assistenzzeiten von Studierenden der Lehramtsfächer an Schulen im Ausland sowie Fortbildungskurse für Lehrkräfte im Ausland. ERASMUS richtet sich an Hochschulen und fördert die Mobilität von Studierenden, Dozenten und anderem Hochschulpersonal. Gefördert werden Auslandsstudium, Auslandspraktikum, Gastdozenturen, die Organisation der Mobilität, Intensivprogramme und vorbereitende Besuche. LEONARDO DA VINCI ist das Programm für die Zusammenarbeit in der beruflichen Aus- und Weiterbildung. Gefördert werden Auslandsaufenthalte in der beruflichen Aus- und Weiterbildung, Projekte zum Transfer von Innovationen, Partnerschaften, Projekte zur Entwicklung von Innovationen, Netzwerke, vorbereitende Besuche und Kontaktseminare. Zielgruppen sind Einrichtungen der beruflichen Bildung wie berufsbildende Schulen, außer- und überbetriebliche Bildungsstätten, Unternehmen, Sozialpartner und ihre Organisationen, Berufsverbände und Kammern. GRUNDTVIG ist das Programm für die allgemeine Erwachsenenbildung. Das Programm steht allen Einrichtungen der Erwachsenenbildung in den Teilnehmerstaaten offen. Dies können öffentliche Institutionen sein wie Behörden, Verwaltungen und Regierungsstellen oder Einrichtungen in öffentlicher oder privater Trägerschaft wie Initiativen, Vereine, Volkshochschulen oder Nichtregierungsorganisationen. Gefördert werden die Mobilität von Beschäftigten der Erwachsenenbildung in Form von individuellen Fortbildungen, Lernpartnerschaften zur Kooperation von Einrichtungen aus verschiedenen Teilnehmerstaaten, Projekte zur Entwicklung, Erprobung und Verbreitung von Produkten und Netzwerke zur Weiterentwicklung von spezifischen Fachgebieten und Themen der Erwachsenenbildung.

In allen Programmen geht es nicht nur um den Erwerb einer Fremdsprache. Aber der Fremdsprachenerwerb ist ein zentrales Ziel dieser Programme. Und auch in der Förderung der Fremdsprachenkompetenz gibt es keine Homogenisierungsbemühung der Europäischen Union, also keine Präferenz für eine bestimmte Fremdsprache. Der Erwerb der kleineren Sprachen wird von der EU genauso gefördert wie das Erlernen der weit verbreiteten Sprachen. 


\section{Transnationales linguistisches Kapital der Bürger der EU}

Wie weit die von der EU geforderte und geförderte Mehrsprachigkeit der Bürger Europas entwickelt ist, ist eine empirische Frage, der ich im Folgenden nachgehen möchte. Mehrsprachigkeit scheint bei einer Konstellation von 23 gleichberechtigten Amtssprachen eine zentrale Voraussetzung zu sein, damit die Menschen am Prozess der Europäisierung der nationalstaatlichen Gesellschaften partizipieren können. Die Kompetenz, eine oder mehrere Sprachen zu sprechen, bezeichne ich hier als linguistisches Kapital. Der Begriff ist von Pierre Bourdieu eingeführt worden (Bourdieu 1992; Bourdieu und Passeron 1971). Ich erläutere zuerst, was Bourdieu unter linguistischem Kapital versteht und ergänze dann dessen Definition um ein weiteres Bestimmungselement.

Bourdieu unterscheidet bekanntlich drei zentrale Kapitalien (vgl. Bourdieu 1982, 1983). ${ }^{4}$ Das ökonomische Kapital umfasst das Einkommen und das Vermögen einer Person. Die Lebensqualität und die Konsummöglichkeiten von Personen hängen entscheidend von der Höhe des ökonomischen Kapitals ab. Das soziale Kapital bezeichnet die Ressource, die Personen aus Beziehungsnetzwerken und Gruppenzugehörigkeiten schlagen können. Je größer das Beziehungsnetzwerk einer Person und je mehr ökonomisches und kulturelles Kapital die anderen Netzwerkmitglieder besitzen, desto höher ist das soziale Kapital einer Person. Das soziale Kapital kann zur Erwirtschaftung von neuen Beziehungen und zum Erwerb der anderen Kapitalien genutzt werden (Bourdieu 1983: 190f). Das kulturelle Kapital ist selbst in drei Unterformen differenziert. Das institutionalisierte kulturelle Kapitel umfasst die Bildung bzw. die Bildungszertifikate, die eine Person durch die Bildungsinstitutionen einer Gesellschaft verliehen bekommen hat. Die Bildungsabschlüsse sichern den Personen den Zugang zu bestimmten Berufen und dadurch den Zugang zu einem bestimmten Einkommen und zu sozialen Netzwerken. Das objektivierte kulturelle Kapital manifestiert sich in Form des Besitzes von Büchern und Gemälden, von kulturellen Artefakten also. Das inkorporierte kulturelle Kapital besteht in der Fähigkeit von Menschen, ästhetische Kriterien zur Beurteilung von „Dingen“ anzuwenden; diese ästhetische Kompetenz steuert die Freizeitaktivitäten von Menschen und prägt ihren Lebensstil.

Das linguistische Kapital ist Teil des inkorporierten kulturellen Kapitals (vgl. Bourdieu und Passeron 1971: 109 ff; Bourdieu 1992). Es besteht in der Fähigkeit, sich in der Hochsprache eines Landes elaboriert mündlich wie schriftlich auszudrücken. Linguistisches Kapital wird ähnlich wie das inkorporierte kulturelle Kapital von Personen eingesetzt, um innerhalb der Klassenstruktur einer Gesellschaft Distinktionsgewinn gegenüber den unteren Klassen zu erzielen. Die Fähigkeit, in der Hochspra-

\footnotetext{
${ }^{4}$ Das symbolische Kapital als vierte Kapitalsorte bezeichnet die soziale Anerkennung der anderen drei Kapitalsorten und dient damit der Legitimierung sozialer Ungleichheit. Ein Bauunternehmer z. B., der über ein großes finanzielles Vermögen verfügt, kann dieses ökonomische Kapital in symbolisches Kapital (Legitimierung durch gesellschaftliche Anerkennung) transferieren, wenn er einen Teil seines Vermögens an eine gemeinnützige Hilfsorganisation spendet.
} 
che eines Landes in einem elaborierten Code zu kommunizieren, eröffnet die Möglichkeit, sich gegenüber der Alltagssprache der unteren Klassen mit ihrer „vulgären Sprache“ (Bourdieu und Passeron 1971: 110) und ihrem restringierten Code abzugrenzen. Vor allem die Ausbildungsinstitutionen prämieren die Fähigkeit, sich in der Hochsprache gewählt artikulieren zu können. Und da die Verfügung über linguistisches Kapital von der Klassenlage von Personen abhängig ist, reproduzieren die Ausbildungsinstitutionen die Klassenstruktur einer Gesellschaft. Welche Sprache nun als Hochsprache gilt und sich gegenüber anderen Sprachen und Dialekten durchgesetzt hat, ist das Resultat eines historischen Prozesses. Bourdieu (1992) hat diesen Prozess für Frankreich rekonstruiert und gezeigt, wie es den Eliten gelungen ist, eine Einheitssprache durchzusetzen und andere Sprachen und Dialekte zu delegitimieren (Bourdieu 1992; vgl. dazu auch Loos 2000; aus der Perspektive eines Historikers vgl. Weber 1976).

So wie die Arbeiten Bourdieus insgesamt im hohen Maße dem methodologischen Nationalismus (Beck und Grande 2004) verhaftet bleiben, indem sie vor allem die Klassenstruktur einer nationalstaatlich verfassten Gesellschaft analysieren, so bleibt auch sein Konzept des linguistischen Kapitals weitgehend auf den Nationalstaat bezogen. Der Begriff des linguistischen Kapitals bezieht sich in erster Linie auf die elaborierte Beherrschung der jeweiligen Hoch- und Amtssprache eines Landes. Im Kontext von Transnationalisierungs- und Europäisierungsprozessen werden aber Kapitalienausstattungen erforderlich, die ein Agieren jenseits des nationalstaatlichen „Containers" (Taylor 1994; Beck 1997) ermöglichen. Die Beherrschung von Fremdsprachen stellt eine solche Ressource dar und wird hier entsprechend als transnationales linguistisches Kapital bezeichnet. Die Fähigkeit, in mehreren Sprachen zu kommunizieren, ist mit zwei Vorteilen verbunden: Sie erleichtert es zum einen erheblich, mit anderen Bürgern anderer Länder in Kontakt zu treten, mit ihnen zu kommunizieren, wirtschaftlich zu handeln, wissenschaftlich zu kooperieren, politische Verhandlungen zu führen, Proteste über die nationalstaatlichen Grenzen hinaus zu organisieren, Liebesbeziehungen einzugehen etc. Peter A. Kraus (2004: 99) bezeichnet dies auch als die instrumentelle Funktion von Sprache, in diesem Fall von Mehrsprachigkeit. Neben diesem instrumentellen Nutzen von Mehrsprachigkeit hat Multilingualität aber auch einen expressiven Nutzen (vgl. Kraus 2004: 100). Akteure, die mehrsprachig sind und dies in Interaktionssituationen zeigen und sich mit dieser Kompetenz inszenieren, definieren sich selbst als Teil einer transnationalen Klasse, die sich mit dieser Kompetenz als "cosmopolitans“ gegenüber den „locals“ abgrenzt und entsprechende symbolische Distinktionsgewinne einfährt.

Die Ausstattung mit linguistischem Kapital versetzt also diejenigen, die über eine Mehrsprachigkeit verfügen, in die Lage, am Transnationalisierungs- und Europäisierungsprozess teilzunehmen und entsprechende transnationale Interaktionsbeziehungen aufzubauen und zugleich den symbolischen Gewinn, Teil einer neuen Elite $\mathrm{zu}$ sein, zu akkumulieren. Insofern ist das transnationale linguistische Kapital eine 
zentrale Ressource der Produktion sozialer Ungleichheit im Kontext von Prozessen der Transnationalisierung.

Die Auswertung einer Eurobarometerbefragung aus dem Jahr 2005 (Eurobarometer 63.4) ermöglicht es zu prüfen, ob und in welchem Maße die Bürger in den 27 Ländern der EU mehrsprachig sind (vgl. für eine Auswertung der Fremdsprachenkompetenz in 15 EU-Ländern Fligstein 2008: 147 ff). Die Frage, mit der die Fremdsprachenkompetenz Bürger erhoben wurde und die auch den folgenden Auswertungen zu Grund liegt, lautet: „Welche Sprache sprechen Sie gut genug, um darin ein Gespräch führen zu können?" Die Umfrage wurde im Mai und Juni 2005 vom Institut TNS im Auftrag der Europäischen Kommission durchgeführt. Interviewt wurden in Form von face-to-face-Interviews Personen, die 15 Jahre und älter sind. Die erreichte Sample-Größe pro Land ist repräsentativ für die Bevölkerung des jeweiligen Landes. Der Datensatz ist über das Zentralarchiv für empirische Sozialforschung in Köln zugänglich. Tabelle 1 gibt die Mehrsprachenkompetenz der Bürger für die 27 Mitgliedsländer der EU wieder. Neben den Kategorien „keine“, „eine“ und „zwei“ Fremdsprachen habe ich den Mittelwert und die Standardabweichung pro Land berechnet.

1) Wie die Ergebnisse zeigen, ist die Europäische Union von dem Ideal, dass alle Bürger mindestens zwei Fremdsprachen sprechen können, weit entfernt. Über die Hälfte der Bürger spricht keine einzige Fremdsprache, ca. ein Viertel spricht zumindest eine Fremdsprache, ein knappes Viertel spricht zwei oder mehr Fremdsprachen.

2) Zugleich zeigen die Berechnungen, dass die Unterschiede zwischen den Ländern in der Ausstattung mit linguistischem Kapital ganz beträchtlich sind. Während in Luxemburg fast jeder Bürger zumindest zweisprachig ist, in Lettland, Malta oder den Niederlanden 90 \% der Bürger zwei und mehr Sprachen beherrschen, liegt die Quote der Menschen, die zumindest eine Fremdsprache spricht, in Ungarn, Portugal, Großbritannien und Spanien bei nur ca. einem Drittel der Bevölkerung.

3) Und schließlich zeigen die Befunde, dass die länderinterne Streuung sehr hoch ist. Die durchschnittliche Standardabweichung beträgt 0.90 bei einer Skala, die von 0 bis 3 reicht. Es gibt also innerhalb jedes Landes Bürger, die über ein hinreichendes linguistisches Kapital verfügen und es gibt Bürger, für die dies nicht zutrifft. 
Tabelle 1: Transnationales linguistisches Kapital in 27 Ländern der EU

\begin{tabular}{|c|c|c|c|c|c|c|}
\hline & $\begin{array}{l}\text { Keine } \\
\text { Fremdspra- } \\
\text { che }\end{array}$ & $\begin{array}{l}\text { Eine } \\
\text { Fremdspra- } \\
\text { che }\end{array}$ & $\begin{array}{l}\text { Zwei } \\
\text { Fremdspra- } \\
\text { chen }\end{array}$ & $\begin{array}{l}\text { Drei und mehr } \\
\text { Fremdsprachen }\end{array}$ & $\begin{array}{l}\text { Mittel- } \\
\text { wert }\end{array}$ & $\mathrm{S}$ \\
\hline EU-27 & $50,7 \%$ & $26,9 \%$ & $15,3 \%$ & $7,1 \%$ & 0,79 & 0,95 \\
\hline Ungarn & $73,9 \%$ & $16,5 \%$ & $7,9 \%$ & $1,8 \%$ & 0,38 & 0,71 \\
\hline Großbritannien & $70,0 \%$ & $20,3 \%$ & $7,4 \%$ & $2,3 \%$ & 0,42 & 0,73 \\
\hline Portugal & $69,9 \%$ & $14,3 \%$ & $11,0 \%$ & $4,8 \%$ & 0,51 & 0,87 \\
\hline Spanien & $64,2 \%$ & $23,4 \%$ & $9,1 \%$ & $3,3 \%$ & 0,52 & 0,79 \\
\hline Rumänien & $61,7 \%$ & $21,1 \%$ & $12,9 \%$ & $4,3 \%$ & 0,60 & 0,87 \\
\hline Italien & $61,0 \%$ & $26,6 \%$ & $10,2 \%$ & $2,3 \%$ & 0,54 & 0,77 \\
\hline Irland & $60,6 \%$ & $24,9 \%$ & $11,8 \%$ & $2,7 \%$ & 0,57 & 0,80 \\
\hline Frankreich & $55,8 \%$ & $27,8 \%$ & $12,5 \%$ & $3,9 \%$ & 0,64 & 0,84 \\
\hline Bulgarien & $55,0 \%$ & $28,6 \%$ & $12,4 \%$ & $4,0 \%$ & 0,65 & 0,85 \\
\hline Polen & $52,5 \%$ & $25,9 \%$ & $12,0 \%$ & $9,6 \%$ & 0,79 & 0,99 \\
\hline Griechenland & $51,3 \%$ & $32,0 \%$ & $13,3 \%$ & $3,4 \%$ & 0,69 & 0,83 \\
\hline Deutschland & $43,8 \%$ & $35,0 \%$ & $17,6 \%$ & $3,6 \%$ & 0,81 & 0,85 \\
\hline Tschechien & $42,1 \%$ & $30,8 \%$ & $18,7 \%$ & $8,3 \%$ & 0,93 & 0,97 \\
\hline Österreich & $40,9 \%$ & $33,6 \%$ & $15,1 \%$ & $10,4 \%$ & 0,95 & 0,99 \\
\hline Finnland & $38,8 \%$ & $22,7 \%$ & $20,4 \%$ & $18,2 \%$ & 1,12 & 1,13 \\
\hline Zypern & $33,3 \%$ & $49,9 \%$ & $12,5 \%$ & $4,4 \%$ & 0,88 & 0,79 \\
\hline Slowakei & $33,1 \%$ & $27,9 \%$ & $21,0 \%$ & $18,0 \%$ & 1,24 & 1,10 \\
\hline Belgien & $28,4 \%$ & $22,8 \%$ & $24,6 \%$ & $24,2 \%$ & 1,45 & 1,14 \\
\hline Estland & $16,0 \%$ & $38,6 \%$ & $29,0 \%$ & $16,5 \%$ & 1,46 & 0,95 \\
\hline Schweden & $13,6 \%$ & $40,4 \%$ & $27,4 \%$ & $18,6 \%$ & 1,51 & 0,94 \\
\hline Dänemark & $13,5 \%$ & $25,2 \%$ & $34,5 \%$ & $26,7 \%$ & 1,74 & 1,00 \\
\hline Litauen & $12,5 \%$ & $43,9 \%$ & $31,1 \%$ & $12,6 \%$ & 1,44 & 0,86 \\
\hline Slowenien & $12,0 \%$ & $27,1 \%$ & $33,8 \%$ & $27,2 \%$ & 1,76 & 0,98 \\
\hline Niederlande & $9,0 \%$ & $21,1 \%$ & $43,6 \%$ & $26,2 \%$ & 1,87 & 0,90 \\
\hline Malta & $7,6 \%$ & $34,2 \%$ & $40,4 \%$ & $17,8 \%$ & 1,68 & 0,85 \\
\hline Lettland & $7,0 \%$ & $51,8 \%$ & $32,9 \%$ & $8,3 \%$ & 1,42 & 0,74 \\
\hline Luxemburg & $1,2 \%$ & $4,2 \%$ & $27,2 \%$ & $67,5 \%$ & 2,61 & 0,63 \\
\hline
\end{tabular}

$\mathrm{N}=26.520$

Interpretiert man die Fähigkeit, mehrere Sprachen zu sprechen als eine zentrale Ressource, die es erlaubt, an Transnationalisierungs- und Europäisierungsprozessen teilzunehmen, dann zeigt sich, dass diese Ressource sehr ungleich verteilt ist. Dies bringt für diejenigen, die über eine hinreichende linguistische Kapitalausstattung verfügen Vorteile, für die anderen Nachteile mit sich. Mehrsprachigkeit erleichtert es, in anderen Ländern zu studieren, zu arbeiten, Kontakte zu Bekannten, Freunden und potentiellen Partner zu knüpfen; es erleichtert Firmen bzw. ihren Mitarbeitern, sich neue Märkte zu erschließen, es erleichtert Wissenschaftlern, ihre Erkenntnisse zu distribuieren und damit ihre eigene Wertschätzung zu erhöhen. Insofern ist der aus der Ökonomie entlehnte Begriff des transnationalen linguistischen Kapitals durchaus berechtigt, weil es sich um einen Produktionsfaktor handelt, dessen Verfügung die eigene Rendite zu steigern vermag und damit auch eine Ressource der Herstellung von Ungleichheit ist. 


\section{Erklärung der Unterschiede in der Verfügung über transnationales linguisti- sches Kapital}

Wir hatten gesehen, dass die Kompetenz zur Mehrsprachigkeit sowohl zwischen den Ländern als auch innerhalb der Länder recht unterschiedlich ausfällt. Ich versuche im Folgenden, die Unterschiede in der Verfügung über transnationales linguistisches Kapital zu erklären und unterscheide dabei zwischen Faktoren, die auf der Individualebene und Faktoren, die auf der Ebene der Länder zu lokalisieren sind. Die genaue Operationalisierung der im Folgenden genauer erläuterten Variablen findet sich im Anhang. Ich beginne mit der Explikation von Erklärungsfaktoren, die sich aus der Theorie Bourdieus ergeben und auf der Individualebene verortet sind.

\subsection{Klassenlage und institutionalisiertes kulturelles Kapital}

Die Bourdieusche Theorie ist eine moderne Klassentheorie; sie erklärt Ungleichheit mit Rückgriff auf die Klassenstruktur einer Gesellschaft. Auch wenn Bourdieu die eigene Theorie nicht zur Erklärung der Verfügung über transnationales linguistisches Kapital in Anschlag gebracht hat, kann man sie problemlos auf diesen Fall applizieren. Die Verfügung über linguistisches Kapital ergibt sich dann aus der Klassenlage einerseits und der hinter den Klassen gelagerten relevanten Kapitalausstattung.

a) Klassenlagen/Klassenfraktionen und Berufsposition: Die Klassenstruktur einer Gesellschaft ergibt sich aus der Aggregation der Kapitalien, über die Personen verfügen und der Zuordnung der Personen mit der gleichen Kapitalausstattung zu ein und derselben Klasse. Bourdieu unterscheidet bekanntlich drei Klassen, die sich im Niveau des Kapitalvolumens voneinander unterscheiden (die Oberklasse, die Mittelklasse und die Unterklasse). Innerhalb der drei Klassen sind die Klassenfraktionen platziert, die durch eine unterschiedliche Zusammensetzung von kulturellem und ökonomischem Kapital gekennzeichnet sind (Bourdieu 1982, 1983). Die Klassen und die Klassenfraktionen werden von Bourdieu nicht nur abstrakt benannt, sondern mit der Angabe von konkreten Berufspositionen genauer bezeichnet. Dies ist für unsere empirische Analyse wichtig, weil der Datensatz es ermöglicht, auf die erhobenen Berufspositionen zurückzugreifen. In der Oberklasse gibt es eine Klassenfraktion mit viel kulturellem Kapital und eine Gruppe mit wenig kulturellem Kapital. Das Besitzbürgertum, bestehend vor allem aus den Selbständigen, verfügt über hohes ökonomisches und im Verhältnis dazu über wenig kulturelles Kapital. Beim Bildungsbürgertum liegt ein umgekehrtes Verhältnis vor: hier dominiert das kulturelle Kapital vor dem Ökonomischen. Zum Bildungsbürgertum zählt Bourdieu vor allem die Professoren und die anderen akademischen Berufe. Die Mittelklasse oder das Kleinbürgertum besteht aus den Inhabern mittlerer Berufspositionen, vor allem aus den mittleren Angestellten. Das Kleinbürgertum ist intern weiter differenziert: das absteigende Kleinbürgertum besitzt wenig oder weniger werdendes ökonomisches und kulturelles Kapital; über ein mittleres Volumen der beiden Kapitalsorten kann das exekutive 
Kleinbürgertum verfügen und als Komplementärklasse zur neuen Bourgeoisie existiert, entsprechend mit mittlerem Kapitalvolumen ausgestattet, das neue Kleinbürgertum. Die Unterklasse, bzw. die Arbeiter- und Bauernschaft wird von Bourdieu nicht weiter in Klassenfraktionen differenziert. Zur Arbeiterschaft gehören die gering gebildeten und manuell tätigen Berufe.

Der Datensatz enhält keine Einkommens- und Vermögensfragen, so dass eine Operationalisierung des ökonomischen Kapitals unmittelbar nicht möglich ist. Die Interviewten wurden aber nach ihrer Berufsposition befragt und diese werden von Bourdieu zur Beschreibung der Klassen und Klassenfraktionen benutzt. Wenn Interviewte zum Zeitpunkt der Befragung nicht erwerbstätig waren - sei es, weil sie zu diesem Zeitpunkt im Haushalt tätig, verrentet oder arbeitslos waren - dann wurden sie nach ihrer früheren Berufsposition gefragt. In einem ersten Schritt wurden die Anworten auf die aktuelle und die frühere Berufsposition miteinander fusioniert. Aus den verschiedenen Kategorien wurden dann folgende Berufsgruppen gebildet.

* Angestellte oder selbständige „Professionals“: Darunter fallen Ärzte, Architekten, Rechtsanwälte, Hochschullehrer etc.

* Höhere und mittlere Leitungskräfte: Darunter fallen Direktoren und Manager, Abteilungsleiter, Techniker, Lehrer etc.

* Selbständige: Erfasst werden hier Geschäfts- und Firmenbesitzer, selbständige Handwerker etc.

* Mittlere Angestellte und Facharbeiter.

* Ungelernte Arbeiter und Angestellte.

Für die Regressionsanalysen wurde aus diesen Berufsgruppen jeweils eine dichotome Variable gebildet. Die ungelernten Arbeiter und Angestellte bilden die Referenzgruppe für die Analysen. Ich gehe erstens davon aus, dass alle Berufsgruppen im Vergleich zu der Referenzgruppe der ungelernten Arbeiter und Angestellten, die Bourdieu dem Proletariat zuordnet, im höheren Maße über linguistisches Kapital verfügen. Ich vermute zweitens, dass die Mittelklasse der Angestellten im geringeren Maße über linguistisches Kapital verfügt als die Selbständigen, die Leitungskräfte und die Professionals. Schließlich kann man vermuten, dass es eine Differenz gibt zwischen den Professionals und den Leitungskräften auf der einen Seite und den Selbständigen auf der anderen. Alle drei Gruppen gehören zur Oberschicht, bilden aber zwei unterschiedliche Fraktionen innerhalb der Oberklasse. Die Selbständigen sind diejenigen, die über viel ökonomisches Kapital, aber über relativ wenig kulturelles Kapital verfügen. ${ }^{5}$ Für die anderen beiden Gruppen gilt die umgekehrte Kapitalstruktur. Entsprechend vermute ich, dass die Selbständigen im Vergleich zu den Professionals und den Leitungskräften im schwächeren Maße über eine transnationale linguistische Kapitalausstattung verfügen.

${ }^{5}$ Da die Kategorie nicht nur Unternehmer sondern auch "kleine" Selbständige wie Handwerker, die Bourdieu eher dem Kleinbürgertum zuordnen würde, enthält, ist die Operationalisierung etwas unscharf. 
b) Institutionalisiertes kulturelles Kapital: Das institutionalisierte kulturelle Kapitel umfasst die Bildung bzw. die Bildungszertifikate, die eine Person durch die Bildungsinstitutionen einer Gesellschaft verliehen bekommen hat. Bildungsabschlüsse in 27 Ländern mit unterschiedlichen Bildungssystemen zu erheben, so dass die Bildungszertifikate miteinander vergleichbar sind, ist nicht einfach. Der Eurobarometer enthält aber eine Variable, die einen Vergleich der Bildungszertifikate trotz der Unterschiede in den Bildungssystemen ermöglicht. Die Interviewten wurden gefragt, wie alt sie waren, als sie ihre Ausbildung beendet haben. Je älter ein Befragter zum Zeitpunkt der Beendigung seiner Ausbildung, desto höher ist sein Bildungsabschluss und desto höher ist sein institutionalisiertes kulturelles Kapital. Mit Bourdieu kann man davon ausgehen, dass das institutionalisierte kulturelle Kapital einen positiven Einfluss auf die Verfügung über transnationales linguistisches Kapital hat, da Fremdsprachen in der Regel über die Ausbildungsinstitutionen vermittelt werden. ${ }^{6}$ Je länger Menschen in den Institutionen verweilen, desto höher wird ihre Verfügung über linguistisches Kapital sein. Neben Bildung und Klassenlage habe ich das Alter des Befragten als Kontrollvariable in die Analyse eingefügt.

\subsection{Länderspezifische Kontextfaktoren}

Individuen sind nicht nur in die Klassenstruktur ihres jeweiligen Landes eingebunden, die Länder selbst unterscheiden sich voneinander durch verschiedene Merkmale, die für den Erwerb der Mehrsprachigkeit eher förderlich bzw. eher hinderlich sein können.

a) Modernisierung der Gesellschaft: Die 27 Länder unterscheiden sich zum einen im Grad der Modernisierung. Wir wissen bis heute nicht genau, welche Faktoren eine Modernisierung befördert haben und wie die Kausalbeziehungen zwischen den verschiedenen Faktoren zu bestimmen sind. Das Ergebnis des Prozesses der Modernisierung ist aber die Entstehung einer Gesellschaft, die durch ein Set von Merkmalen beschreibbar sind, die zusammen ein Syndrom bilden (vgl. Norris 2002: 20ff.). Daniel Bell $(1979,1996)$ unterscheidet zwei Phasen des Modernisierungsprozesses. Modernisierung im Sinne von Industrialisierung meint, dass die industrielle Produktion von Gütern der dominante Produktionsbereich wird und Fabriken und formale Organisationen die dominanten Produktionseinheiten werden, Güter und Dienstleistungen werden über Märkte vermittelt und distribuiert, der Grad der Technisierung der Produktion ist hoch, das Bildungsniveau steigt an, die Urbanisierung ebenfalls. Die zweite Phase der Modernisierung bezeichnet Bell (1979) als Postindustrialisierung. Postindustrialisierung ist mit einer Bedeutungszunahme des Dienstleistungssektors verbunden, so dass dieser zum dominanten Produktionsbereich wird. Technik und Wissenschaftsentwicklung gewinnen zunehmend an Bedeutung, das Niveau

\footnotetext{
${ }^{6}$ Die durch die Bildungsinstitutionen vermittelte Fremdsprachenkompetenz kann dann wiederum zu Erhöhung des institutionalisierten kulturellen Kapitals in Form von Bildungszertifikaten führen.
} 
der Bildung einer Gesellschaft steigt erheblich. Die Zunahme der Bedeutung von Bildung im Kontext des Modernisierungsprozesses manifestiert sich in einer Vielzahl von Indikatoren: dem Rückgang der Analphabetenrate, der Zunahme der Verweildauer von Personen im Bildungssystem, der Zunahme der Quoten von Personen eines Jahrgangs mit höheren Bildungsabschlüssen etc. Ein Teil der Verbesserung der Bildung im Kontext von Modernisierungsprozessen ist die Verbesserung der Ausbildung der Fremdsprachenkompetenz. Insofern kann man vermuten, dass das Niveau der Modernität eines Landes einen Einfluss auf die Fremdsprachenkompetenz seiner Bürger hat. Menschen, die in einem Land leben, das einen hohen Grad der Modernität erreicht hat, werden mehr Fremdsprachen können als Menschen, die in einem Land leben, das geringer modernisiert ist. Zur Messung des Grades der Modernisierung benutzen wird den „Human Development Index“ (HDI) aus dem Jahr 2004. In den HDI gehen das reale Bruttosozialprodukt pro Einwohner, das Bildungsniveau und die durchschnittliche Lebenserwartung ein.

b) Größe eines Landes: Je kleiner ein Land ist, desto größer ist seine internationale Vernetzung. Je größer die internationale Vernetzung eines Landes, desto besser ist die Fremdsprachenkompetenz seiner Bürger. Für diesen Zusammenhang lassen sich in der Literatur verschiedene Argumente finden. Ich orientiere mich im Folgenden vor allem an den Überlegungen von Hans Geser (1992). Aus der Geometrie kann man lernen, dass der Grenzumfang einer Fläche im degressiven Verhältnis zu seiner Innenfläche wächst. Dies bedeutet, dass ein kleines Land (Innenfläche) überdurchschnittlich viel Grenzfläche mit seinen Nachbarländern hat. Dieser Tatbestand allein erhöht die Wahrscheinlichkeit von Außenkontakten. Wenn das Ausland zugleich durch eine andere Sprache gekennzeichnet ist, dann wirkt sich dies positiv auf den Fremdsprachenerwerb aus. Dies gilt erst recht und besonders für moderne, arbeitsteilige Gesellschaften. Die Wahrscheinlichkeit, einen Kommunikations- und Handelspartner für seine Interessen und Güter zu finden, der selbst Mitglied der eigenen Gesellschaft ist, ist für kleine Gesellschaften deutlich geringer als für große Gesellschaften. Entsprechend sind kleine Gesellschaften in der Regel in einem wesentlich höheren Maße in internationale wirtschaftliche und kommunikative Austauschprozesse integriert als größere Länder. Der Austausch mit anderen Ländern wird wiederum wesentlich erleichtert, wenn die Menschen die Sprache des Auslandes sprechen.

Es gibt noch einen weiteren Grund, warum die Größe eines Landes wahrscheinlich einen Einfluss auf die Fremdsprachenkompetenz der Bürger hat. Menschen lernen eine Fremdsprache nicht nur durch Bildungsinstitutionen und durch unmittelbare Kontakte mit Menschen, die eine andere Sprache sprechen, sondern auch durch die Rezeption medialer Produkte (Bücher, Musik, Filme). Die Übersetzung und Synchronisation von ausländischen Medienprodukten lohnt sich für einen Verlag, einen Filmvertrieb oder für die Radio- und Fernsehanstalten nur, wenn die Menge der Abnehmer der Produkte eine bestimme Größenordnung erreicht. Ist das nicht der Fall, werden die Produkte nicht übersetzt und nur in der Originalsprache distribuiert 
bzw. (bei Filmen und Fernsehsendungen) allein mit Untertiteln versehen ausgestrahlt. Der Anteil der Medienprodukte, die in der Originalsprache distribuiert wird, ist entsprechend in kleinen Ländern deutlich höher als in großen Ländern. Menschen, die fremdsprachige Medienprodukte rezipieren, werden dadurch ihre Fremdsprachenkompetenz verbessern. Die Größe eines Landes habe ich durch die Menge der Einwohnerzahl operationalisiert.

c) Die Dominanz der Muttersprache: In der ökonomischen Theorie des Fremdsprachenerwerbs wird die Wahrscheinlichkeit des Erwerbs einer Fremdsprache aus dem Kommunikationsnutzen einer Sprache abgeleitet (vgl. de Swaan 2001a; 2001b). Der Kommunikationsnutzen des Erwerbs einer Fremdsprache ist gering, wenn Menschen eine Muttersprache sprechen, die von vielen anderen im Ausland auch gesprochen wird, sei es als Muttersprache, sei es als Fremdsprache. Es ist dann wahrscheinlich, dass sie sich in ihrer Muttersprache mit Leuten im Ausland verständigen können, weil diese ihre Sprache sprechen; die Notwendigkeit, selbst eine Fremdsprache zu lernen und zu sprechen, ist also gering. Diese Randbedingung scheint für das Englische zu gelten. Es ist diejenige Sprache, die innerhalb Europas, aber auch innerhalb der Welt, am häufigsten gesprochen wird (Crystal 1998). Nach unserer Berechnung sind es 43 \% der Bürger der 27 Länder der EU, die angeben, Englisch als Mutter- oder als Fremdsprache sprechen zu können. Englisch ist damit die mit Abstand meist verbreitete Sprache in Europa. Entsprechend vermute ich, dass diejenigen, die Englisch als Muttersprache sprechen, über ein geringeres transnationales linguistisches Kapital verfügen als Personen, für die dies nicht gilt.

\section{3 Überprüfung der Hypothesen}

Ich habe zwei verschiedene multivariate Analysen durchgeführt; zum einen eine lineare Regression, in der die Menge der Fremdsprachen, die jemand spricht, die abhängige Variable bildet. Da die Variable rechtsschief verteilt ist (siehe Tabelle 1), habe ich zusätzlich eine logistische Regression berechnet, in der die Frage, ob jemand überhaupt eine Fremdsprache beherrscht oder nicht, die dichotome abhängige Variable bildet. Die Ergebnisse der logistischen und der linearen Regressionsanalysen sind nahezu identisch. Ich konzentriere mich im Folgenden allein auf die Darstellung und Interpretation der linearen Regressionsanalysen. ${ }^{7}$

Tabelle 2 enthält sieben verschiedene Regressionsanalysen, die ich im Folgenden nacheinander interpretiere. ${ }^{8}$

\footnotetext{
${ }^{7}$ Auch die Ergebnisse einer durchgeführten Mehrebenenanalyse unterscheiden sich nicht von den hier präsentierten Befunden.

${ }^{8}$ Die Stichproben der Länder wurden so gewichtet, dass sie der Bevölkerungsanzahl der jeweiligen Länder entsprechen.
} 
Tabelle 2: Erklärung der Verfügung über transnationales linguistisches Kapital (lineare Regressionen)

\begin{tabular}{|c|c|c|c|c|c|c|c|}
\hline & Modell 1 & Modell 2 & Modell 3 & Modell 4 & Modell 5 & Modell 6 & Modell 7 \\
\hline \multicolumn{8}{|l|}{$\begin{array}{l}\text { Klassen und Klas- } \\
\text { senfraktionen }^{1}\end{array}$} \\
\hline $\begin{array}{l}\text { Angestellte oder } \\
\text { selbstständige Pro- } \\
\text { fessionals }\end{array}$ & $0,13^{* * *}$ & $0,11^{* * *}$ & $0,07^{* * *}$ & $0,06^{* * *}$ & $0,07^{* * *}$ & & $0,07^{* * *}$ \\
\hline $\begin{array}{l}\text { Höhere und mittlere } \\
\text { Leitungskräfte }\end{array}$ & $0,16^{* * *}$ & $0,15^{* * *}$ & $0,10^{* * *}$ & $0,10^{* * *}$ & $0,10^{* * *}$ & & $0,10^{* * *}$ \\
\hline Selbstständige & $0,01^{* * *}$ & $0,02^{* * *}$ & $0,03^{* * *}$ & $0,03^{* * *}$ & $0,03^{* * *}$ & & $0,02^{* * *}$ \\
\hline $\begin{array}{l}\text { Mittlere Angestellte } \\
\text { und Facharbeiter }\end{array}$ & $0,06^{* * *}$ & $0,05^{* * *}$ & $0,06^{* * *}$ & $0,05^{* * *}$ & $0,05^{* * *}$ & & $0,04^{* * *}$ \\
\hline $\begin{array}{l}\text { Kulturelles Kapital } \\
\text { (Bildung) }\end{array}$ & & & $0,35^{* * *}$ & $0,34^{* * *}$ & $0,33^{* * *}$ & & $0,32^{* * *}$ \\
\hline Alter & & $-0,22^{* * *}$ & & $-0,07^{* * *}$ & $-0,06^{* * *}$ & & $-0,06^{* * *}$ \\
\hline $\begin{array}{l}\text { Englisch als Mutter- } \\
\text { sprache }\end{array}$ & & & & & $-0,13^{* * *}$ & & $-0,13^{* * *}$ \\
\hline $\begin{array}{l}\text { Modernitätsgrad } \\
\text { eines Landes (HDI) }\end{array}$ & & & & & & $0,10^{* * *}$ & $0,13^{* * *}$ \\
\hline Größe eines Landes & & & & & & $-0,23^{* * *}$ & $-0,21^{* * *}$ \\
\hline $\mathbf{N}$ & 26520 & 26520 & 23562 & 23562 & 23562 & 26520 & 23562 \\
\hline $\mathbf{R}^{2}$ & $3,6 \%$ & $8,5 \%$ & $16,8 \%$ & $17,3 \%$ & $19,0 \%$ & $4,2 \%$ & $22,7 \%$ \\
\hline
\end{tabular}

Angegeben sind die standardisierten Regressionskoeffizienten. ${ }^{*} \mathrm{p}_{\mathrm{t}}<, 05 ;{ }^{* * *} \mathrm{p}_{\mathrm{t}}<, 001,{ }^{1}$ Referenzkategorie bilden die ungelernten Arbeiter.

1. In einem ersten Schritt wurde die Berufsposition der Befragten mit in die Analyse aufgenommen. Es bestätigt sich die Erwartung, dass alle Berufsgruppen im Vergleich zu der Referenzgruppe der ungelernten Arbeiter und Angestellten im höheren Maße über ein höheres linguistisches Kapital verfügen. Vergleicht man die Berufsgruppen untereinander, dann sieht man, dass die Mittelklasse der Angestellten im geringeren Maße mehrsprachig ist als die zwei Gruppen der Oberklasse (Leitungskräfte, Professionals); auch dies entspricht der Bourdieuschen Hypothese. Weiterhin zeigt sich eine Differenz zwischen den Professionals und den Leitungskräften auf der einen Seite und den Selbständigen auf der anderen Seite. Dies deutet darauf hin, dass die beiden Gruppen zwei Fraktionen innerhalb der Oberklasse bilden. Die Selbständigen sind diejenigen, die über viel ökonomisches, aber über relativ wenig kulturelles Kapital und linguistisches Kapital verfügen.

2. Im zweiten, dritten Modell und vierten Modell wurde zusätzlich das institutionalisierte kulturelle Kapital des Befragten in die Analyse einbezogen, jeweils mit und ohne Kontrolle des Alters. Zum einen zeigt sich, dass der Alterseffekt fast vollständig von der Bildung des Befragten ",aufgesaugt" wird. Die jüngeren Befragten sind auch die besser Ausgebildeten und dies erklärt, warum in Modell 2, in dem allein die Al- 
tersvariable, nicht aber die Bildungsvariable enthalten ist, ein starker Effekt vom Alter auf die Mehrsprachigkeit ausgeht. Dieser Einfluss verschwindet fast vollständig, wenn man zusätzlich den Einfluss der Bildung des Befragten berücksichtigt. Wie das Vorzeichen des Koeffizienten zeigt, geht der Effekt in die theoretisch erwartete Richtung: Je höher die Bildung des Befragten, desto besser die Ausstattung mit linguistischem Kapital. Die Berücksichtigung der Bildung reduziert zugleich den Einfluss der Klassenlage auf die Erklärung des linguistischen Kapitals. Schließlich zeigen die Analysen, dass die Berücksichtigung des institutionalisierten kulturellen Kapitals die Erklärungsleistung des Modells von 3,6 \% auf 17,3 \% erhöht. Von der Bildung des Befragten geht also der stärkste Effekt auf die Mehrsprachigkeit aus. Insgesamt werden durch die Analysen die Bourdieuschen Annahmen sehr gut bestätigt. Die Verfügung über linguistisches Kapital wird entscheidend bestimmt durch die Klassenlage und die Kapitalausstattung des Befragten.

3. Die Modelle 5 bis 7 berücksichtigen die Kontextvariablen bei der Erklärung von Mehrsprachigkeit. Modell 5 fügt zuerst die auf der Individualebene erhobene Frage, ob jemand Englisch als Muttersprache spricht, hinzu. Die aufklärte Varianz erhöht sich auf $19 \%$, der Effekt geht in die erwartete Richtung. Diejenigen, die Englisch als Muttersprache sprechen, verfügen über eine geringere Fremdsprachkompetenz. Modell 6 enthält nur die Makrovariablen. Vergleicht man die aufklärte Varianz in Modell 5 mit der in Modell 6, dann sieht man, dass die Kontextvariablen deutlich weniger zur Erklärung beitragen als die Individualvariablen. Die Effekte der beiden unabhängigen Makrovariablen gehen aber in die theoretisch erwartete Richtung: Je modernisierter und je kleiner ein Land ist, desto besser ist die Ausstattung seiner Bevölkerung mit transnationalem linguistischem Kapital. Das letzte Modell schließlich berücksichtigt alle theoretisch begründeten Einflussfaktoren. Wie der $\mathrm{R}^{2}$-Wert von 22,7 \% ausweist, kann man die Fremdsprachenkompetenz mit den unabhängigen Variablen sehr gut voraussagen. Zudem werden alle unsere theoretischen Annahmen bestätigt. Den stärksten Einfluss auf die Mehrsprachigkeit des Befragten haben das institutionalisierte kulturelle Kapital des Befragten und die Größe des Landes, aus dem er kommt.

\section{Bilanz der Befunde}

Der politische Prozess der europäischen Integration hat zu einer Europäisierung der Mitgliedsländer im Sinne einer Erhöhung der transnationalen Austauschbeziehungen und der wechselseitigen Interdependenzen geführt. Ob dieser systemische Prozess der Europäisierung von einer lebensweltlichen Europäisierung im Sinne einer Zunahme der Kommunikation zwischen den Bürgern verschiedener Mitgliedsländer begleitet wird, hängt entscheidend davon ab, ob und in welchem Maße die Bürger miteinander kommunizieren können. Wir hatten gesehen, dass die EU im Unterschied zu den Nationalstaaten keine Politik der sprachlichen Homogenisierung und der Förderung einer Einheitssprache betreibt. Die Europäische Union akzeptiert die Amtssprachen der Mitgliedsländer als ihre eigenen Amtssprachen, so dass in der 
Union 23 Sprachen den Status einer Amtssprache haben; die EU fördert zugleich die Mehrsprachigkeit der Bürger Europas.

Unter den Bedingungen von Transnationalisierungs- und Europäisierungsprozessen einerseits und einer Politik der Akzeptanz eines multilingualen Europas ist die Fähigkeit, eine oder mehrere Fremdsprachen zu sprechen eine wichtige Ressource zur Partizipation am Europäisierungsprozess. Die Ausstattung mit transnationalem linguistischem Kapital erleichtert es, mit anderen Bürgern anderer Länder in Interaktionsbeziehungen unterschiedlicher Art treten zu können; die Fremdsprachenkompetenz bietet zudem die Möglichkeit, sich als Teil einer neuen transnationalen Klasse darstellen zu können und entsprechende symbolische Distinktionsgewinne zu akkumulieren. Insofern ist das transnationale linguistische Kapital eine zentrale Ressource der Produktion sozialer Ungleichheit im Kontext von Prozessen der Transnationalisierung.

Unsere empirischen Analysen zeigen zum einen, dass es mit der Fremdsprachenkompetenz der Bürger nicht so weit her ist; mehr als 50 \% der Bürger sprechen keine Fremdsprache; deren Partizipationschancen am Europäisierungsprozess sind also sehr eingeschränkt. Die Auswertungen zeigen zum zweiten, dass die Kompetenzen der Mehrsprachigkeit zwischen den Ländern und innerhalb der Länder sehr unterschiedlich verteilt sind, es ähnlich wie bei anderen Kapitalien eine Ungleichheit in der Kapitalausstattung der Bürger gibt. Wir haben deswegen untersucht, wie man diese Ungleichheit erklären kann. Neben der Größe eines Landes und der Frage, ob in dem Land Englisch als Muttersprache gesprochen wird, hat die Ungleichheit zwischen den Ländern und die Ungleichheit innerhalb der Länder einen entscheidenden Einfluss auf die Ausstattung mit transnationalem linguistischem Kapital: Je modernisierter ein Land ist, desto wahrscheinlicher ist es, dass die Bürger über eine bessere linguistische Kapitalausstattung verfügen. Und es sind die oberen Klassen, die über eine gute Bildung verfügen, die für eine Teilhabe am Prozess der europäischen Integration gut gerüstet sind, weil sie eine entsprechende Fremdsprachenkompetenz haben. Das Projekt Europa ist neben allen anderen Dingen eben auch ein Klassenprojekt (Fligstein 2008). Und es sind die oberen Klassen, die für den Europäisierungsprozess besser ausgestattet sind und von diesem am meisten profitieren, die sich mit dem Projekt Europas auch am stärksten identifizieren, die also die Kerneuropäer darstellen (Fligstein 2008: 145).

Zugleich muss man aber in Rechnung stellen, dass nicht nur der Prozess der Europäisierung nationalstaatlich verfasster Gesellschaften eine enorme Dynamik aufweist, sondern auch der Fremdsprachenerwerb der Bürger. Während in der Gruppe der älter als 65-Jährigen 58,4 \% angeben, keine Fremdsprache zu sprechen, beträgt der Anteil in der Gruppe der 15-24-Jährigen nur noch 22,8 \%. Insofern mögen sich die Unterschiede in der Fremdsprachenkompetenz zwischen und innerhalb der Länder im Zeitverlauf verringern, wenn es den Ländern gelingt, sich weiter zu modernisieren, das Bildungsniveau insgesamt anzuheben und die Klassenunterschiede abzuschwächen. 


\section{Literatur}

Ambrosius, Gerold, 1996: Wirtschaftsraum Europa. Vom Ende der Nationalökonomien. Frankfurt a.M.: Fischer.

Beck, Ulrich, 1997: Was ist Globalisierung? Irrtümer des Globalismus - Antworten auf Globalisierung. Frankfurt a.M.: Suhrkamp.

Beck, Ulrich, und Edgar Grande, 2004: Das kosmopolitische Europa. Frankfurt a.M.: Suhrkamp.

Beisheim, Marianne, Sabine Dreher, Gregor Walter, Bernhard Zangl und Michael Zürn, 1999: Im Zeitalter der Globalisierung? Thesen und Daten zur gesellschaftlichen und politischen Denationalisierung. Baden-Baden: Nomos.

Bell, Daniel, 1979: Die Zukunft der westlichen Welt. Kultur und Technologie im Widerstreit. Frankfurt a.M.: Fischer.

Bell, Daniel, 1996: Die nachindustrielle Gesellschaft. Frankfurt a.M.: Campus.

Bourdieu, Pierre, 1982: Die feinen Unterschiede. Kritik der gesellschaftlichen Urteilskraft. Frankfurt a.M.: Suhrkamp.

Bourdieu, Pierre, 1983: Ökonomisches Kapital, kulturelles Kapital, soziales Kapital. S. 183-198 in: Reinhard Kreckel (Hg.): Soziale Ungleichheit. Sonderband 2 der Sozialen Welt. Göttingen: Otto Schwarz \& Co.

Bourdieu, Pierre, und Jean-Claude Passeron, 1971 : Die Illusion der Chancengleichheit. Stuttgart: Ernst Klett Verlag.

Bourdieu, Pierre, 1992: Language and Symbolic Power. Cambridge: Polity Press.

Crystal, David, 1998: English as a Global Language. Cambridge: Canto.

De Swaan, Abram, 1995: Die soziologische Untersuchung der transnationalen Gesellschaft. Journal für Sozialforschung 35: 107-120.

De Swaan, Abram, 1993: The evolving European Language System: A Theory of Communication Potential and Language Competition. International Political Science Review 14(3): 241-256.

De Swaan, Abram, 2001a: The Language Constellation of the European Union. S. 170-181 in: Martin Kohli und Mojca Novak (Hg.): Will Europe Work? Integration, employment and the social order. London/New York: Routledge.

De Swaan, Abram, 2001b: Words of the World - The Global Language System. Cambridge: Polity Press.

Delhey, Jan, 2004: Nationales und transnationales Vertrauen in der Europäischen Union. Leviathan 32: $15-45$.

Delhey, Jan, 2005: Das Abenteuer der Europäisierung. Überlegungen zu einem soziologischen Begriff europäischer Integration und zur Stellung der Soziologie zu den Integration Studies. Soziologie 34: 7-27.

Deutsch, Karl W., 1953: Nationalism and social communication. An inquiry into the foundations of nationality. Cambridge: University Press.

Europäische Wirtschaftsgemeinschaft (EWG), 1958: Verordnung Nr. 1 zur Regelung der Sprachenfrage für die Europäische Wirtschaftsgemeinschaft, http://eurlex.europa.eu/LexUriServ/LexUriServ.do?uri=CONSLEG:1958R0001:20070101:DE:PDF (Zugriff: 03.12.2008).

Fligstein Neil, 2008: Euroclash. The EU, European Identity, and the Future of Europe. Oxford University Press.

Fligstein, Neil, und Frederic Merand, 2002: Globalization or Europeanization? Changes in the European Economy, 1980- 2000. Acta Sociologica 45: 7-22.

Fligstein, Neil, und Alec Stone Sweet, 2002: Constructing Polities and Markets: An Institutionalist Account of European Integration. American Journal of Sociology 107: 1206-1243.

Generaldirektion Bildung und Kultur, 2008: Programm für lebenslanges Lernen. http://www.lebenslanges-lernen.eu (Zugriff: 24.11.08). 
Generaldirektion Übersetzung, 2008:

http://ec.europa.eu/dgs/translation/navigation/faq/faq_facts_de.htm (Zugriff: 03.12.08).

Gerhards, Jürgen, und Holger Lengfeld, 2008: Support for European Union environmental policy by citizens of EU-member and accession states. Comparative Sociology 7: 215-241.

Gerhards, Jürgen, und Jörg Rössel, 1999: Zur Transnationalisierung der Gesellschaft der Bundesrepublik. Entwicklungen, Ursachen und mögliche Folgen für die europäische Integration. Zeitschrift für Soziologie, 28(5): 325-344.

Gerhards, Jürgen, Sylvia Kämpfer und Mike Schäfer, 2009: Gender Equality: The EU Script and its Support in the Member States. Sociology (im Erscheinen).

Geser, Hans, 1992: Kleinstaaten im internationalen System. Kölner Zeitschrift für Soziologie und Sozialpsychologie 44: 627-654.

Haarmann, Harald, 1993: Die Sprachenwelt Europas. Geschichte und Zukunft der Sprachnationen zwischen Atlantik und Ural. Frankfurt a.M./New York: Campus.

Hirst, Paul, und Graham Thompson, 1998: Globalisierung? Internationale Wirtschaftsbeziehungen, Nationalökonomien und die Formierung von Handelsblöcken. S. 85-133 in: Ulrich Beck (Hg.): Politik der Globalisierung. Frankfurt a.M.: Suhrkamp.

Kaelble, Hartmut, 2005: Eine europäische Gesellschaft? S. 299-330 in: Gunnar Folke Schuppert, Ingolf Pernice und Ulrich Haltern (Hg.): Europawissenschaft. Baden Baden: Nomos.

Kaelble, Hartumut, 2007: Sozialgeschichte Europas. 1945 bis zur Gegenwart. München: Beck.

Kantner, Cathleen, 2004: Kein modernes Babel. Kommunikative Voraussetzungen europäischer Öffentlichkeit. Wiesbaden: Verlag für Sozialwissenschaften.

Kraus, Peter A., 2004: Europäische Öffentlichkeit und Sprachpolitik - Integration durch Anerkennung. Frankfurt a.M./New York: Campus.

Lepsius, M. Rainer, 1990: Der europäische Nationalstaat: Erbe oder Zukunft. S. 256-268 in: M. Rainer Lepsius (Hg.): Interessen, Ideen, Institutionen. Opladen. Westdeutscher Verlag.

Loos, Eugène, 2000: Language Choice, Linguistic Capital and Symbolic Denomination in the European Union. Language Problems \& Language Planning 24(1): 37-53.

Mann, Michael, 2001: The Dark Side of Democracy. The Modern Tradition of Ethnic and Political Cleaning. New Left Review 235: 18-45.

Mau, Steffen, und Jan Mewes 2007: Transnationale soziale Beziehungen. Eine Kartographie der deutschen Bevölkerung. Soziale Welt 58(2): 203-222.

Mau, Steffen, 2007: Transnationale Vergesellschaftung. Die Entgrenzung sozialer Lebenswelten. Frankfurt a.M.: Campus.

Mickel, Wolfgang, und Jan Bergmann, 2005: Handlexikon der Europäischen Union. 3. Auflage. BadenBaden: Nomos.

Norris, Pippa, 2002: Democratic Phoenix. Cambridge: University Press.

Ozvalda, Margit, 2005: Sprach- und Sprachenpolitik der EU: Planung, Praxis und Prognosen. EU Working Papers 2: 61-80.

Peterson, John, und Michael Shackleton (Hg.), 2006: The Institutions oft the European Union. 2. Auflage. Oxford: University Press.

Pries, Ludger, 2008: Die Transnationalisierung der sozialen Welt. Sozialräume jenseits von Nationalgesellschaften. Frankfurt a.M.: Suhrkamp.

Rokkan, Stein, 1999: State Formation, Nation Building, and Mass politics in Europe. Oxford: University Press.

Schumann-Hitzler, Gerhard, und Martina Ostarek, 2005: Europahandbuch 2005/2006 - Institutionen, Organisationen, Ansprechpartner. 9. Auflage. Köln: Heymann.

Taylor, Peter J., 1994: The State As Container. Territoriality in the Modern World-System. Progress in Human Geography, 18(2): 151-162.

Trabant, Jürgen, 2008: Was ist Sprache? München: Beck.

Verwiebe, Roland, 2004: Transnationale Mobilität innerhalb Europas. Eine Studie zu den sozialstrukturellen Effekten der Europäisierung. Berlin: edition sigma. 
Vobruba, Georg, 2001: Integration und Erweiterung. Europa im Globalisierungsdilemma. Wien: Passagen.

Vobruba, Georg, 2005: Die Dynamik Europas. Wiesbaden: VS Verlag für Sozialwissenschaften.

Vobruba, Georg, 2008: Die Entwicklung der Europasoziologie aus der Differenz national/europäisch. Berliner Journal für Soziologie 18 (1): 32-51

Weber, Eugen, 1976: Peasants into Frenchmen. The Modernization of Rural France, 1870-1914. Stanford: University Press.

Weidenfeld, Werner (Hg.), 2006: Europa von A bis Z. 9. Auflage. Baden-Baden: Nomos.

Zürn, Michael, 1998: Regieren jenseits des Nationalstaates. Globalisierung und Denationalisierung als Chance. Frankfurt: Suhrkamp.

\section{Internetquellen}

Bundesregierung (Europa-Lexikon):

http://www.bundesregierung.de/Content/DE/Lexikon/EUGlossar/EUGlossar.html?init_entryId= 36304\&init_alpha=S\#doc36304bodyText1

Europäischer Gerichtshof:

http://curia.europa.eu/de/instit/presentationfr/index_cje.htm

Europäischer Rat: http://consilium.europa.eu/cms3_fo/showPage.asp?id=1255\&lang=DE\&mode=g

Europäisches Parlament (Geschäftsordnung):

http://www.europarl.europa.eu/sides/getDoc.do?pubRef=-//EP//TEXT+RULES-EP+20081022+RULE-

138+DOC+XML+V0//DE\&language $=$ DE\&navigationBar=YES

Europäisches Parlament (Übersetzungsdienst):

http://www.europarl.europa.eu/parliament/public/staticDisplay.do?id=155\&pageRank=3\&language= $\mathrm{DE}$

Übersetzungszentrum für die Einrichtungen der Europäischen Union:

http://europa.eu/agencies/community_agencies/cdt/index_de.htm 


\begin{tabular}{|c|c|c|c|}
\hline \multicolumn{4}{|c|}{ Anhang 1: $\quad$ Beschreibung der Variablen } \\
\hline Variable & Ausprägungen & Beschreibung & Datenquelle \\
\hline $\begin{array}{l}\text { Transnationales } \\
\text { linguistisches Kapi- } \\
\text { tal }\end{array}$ & $0-3$ & $\begin{array}{l}\text { Menge der gesprochenen Fremdsprachen; Ko- } \\
\text { dierung } 0 \text { „,keine“ } 1 \text { „eine Fremdsprache“ } 2 \\
\text { „zwei Fremdsprachen“ } 3 \text { „drei oder mehr } \\
\text { Fremdsprachen“ }\end{array}$ & EB 63.4 \\
\hline $\begin{array}{l}\text { Klassen } \\
\text { - Angestellte/ } \\
\text { selbstständige Pro- } \\
\text { fessionals, } \\
\text { - Höhere/mittlere } \\
\text { Leitungskräfte, } \\
\text { - Selbstständige, } \\
\text { - Mittlere Angestell- } \\
\text { te und Facharbeiter }\end{array}$ & $0-1$ & $\begin{array}{l}\text { Dummyvariablen gebildet aus derzeitiger und } \\
\text { früherer Beschäftigung; Kodierung } 0 \text { „nein“ } 1 \\
\text {,ja“. Referenzkategorie: Ungelernte Arbeiter } \\
\text { und Angestellte. }\end{array}$ & EB 63.4 \\
\hline $\begin{array}{l}\text { Institutionalisiertes } \\
\text { kulturelles Kapital }\end{array}$ & $0-65$ & $\begin{array}{l}\text { Alter bei Ausbildungsende; Kodierung } 0 \text { „, noch } \\
\text { studierend“, } 98 \text { „Keine (Vollzeit)Ausbildung, } 99 \\
\text { „Weiß nicht“" } \\
\text { Operationalisierung: Kategorie 0, } 98 \text { und } 99= \\
\text { Missing }\end{array}$ & EB 63.4 \\
\hline Alter & & $\begin{array}{l}\text { Alter zum Zeitpunkt der Befragung; } \\
\text { von } 15 \text { bis } 97 \text { Jahren }\end{array}$ & EB 63.4 \\
\hline $\begin{array}{l}\text { Englisch als Mut- } \\
\text { tersprache }\end{array}$ & $0-1$ & $\begin{array}{l}\text { Dummyvariable; Kodierung } 0 \text { „,nicht angege- } \\
\text { ben“ } 1 \text { „, angegeben“ }\end{array}$ & EB 63.4 \\
\hline $\begin{array}{l}\text { Modernisierungs- } \\
\text { grad }\end{array}$ & & $\begin{array}{l}\text { Human Development Index zusammengesetzt } \\
\text { aus Alphatisierungsgrad, Einschulungsrate, } \\
\text { Lebenserwartung und BIP pro Kopf; } \\
\text { von Rumänien }(0,805) \text { bis Irland }(0,956)\end{array}$ & $\begin{array}{l}\text { Human Deve- } \\
\text { lopment Re- } \\
\text { port } 2006\end{array}$ \\
\hline Größe eines Landes & & $\begin{array}{l}\text { Anzahl der Einwohner pro Land; } \\
\text { von Luxemburg (450.000) bis Deutschland } \\
(82.534 .000)\end{array}$ & \begin{tabular}{|l} 
Datenreport \\
2006
\end{tabular} \\
\hline
\end{tabular}

